\title{
Le cerf chasseur et le maïs agriculteur : l'identité des divinités dans les mitote náyeri
}

\section{Margarita Valdovinos}

\section{OpenEdition}

\section{Journals}

Édition électronique

URL : https://journals.openedition.org/jsa/9673

DOI : $10.4000 /$ jsa.9673

ISSN : 1957-7842

Éditeur

Société des américanistes

Édition imprimée

Date de publication : 15 juillet 2008

Pagination : 231-250

ISSN : 0037-9174

\section{Référence électronique}

Margarita Valdovinos, "Le cerf chasseur et le maïs agriculteur : l'identité des divinités dans les mitote náyeri », Journal de la Société des américanistes [En ligne], 94-1 | 2008, mis en ligne le 10 juin 2013, consulté le 03 septembre 2022. URL : http://journals.openedition.org/jsa/9673 ; DOI : https:// doi.org/10.4000/jsa.9673 


\title{
LE CERF CHASSEUR ET LE MAÏS AGRICULTEUR : L'IDENTITÉ DES DIVINITÉS DANS LES MITOTES NÁYERI
}

\author{
Margarita VALDOVINOS *
}

Cet article montre comment se constitue l'identité des divinités náyeri au cours des cérémonies agricoles nommées mitote. Après l'analyse des charges rituelles censées personnifier les divinités et des liens existant entre elles, seront abordés d'autres éléments qui, souvent écartés de la réflexion sur les représentations des divinités, s'avèrent être également des composants essentiels : les objets et les chants. À partir des actions et des rôles assumés par les personnifications des divinités, il s'agit ici de dégager la complexité de leur présence dans le rituel et les effets que cela entraîne chez les exécutants du mitote. [Mots-clés : rituel, Cora (Náyeri), charges rituelles, maïs, cerf, agriculture, chasse, divinités, identité, Nayarit.]

The hunting deer and the agriculturalist maize. The deities' identity in the náyeri's mitote. This article shows how the identity of the náyeri deities is constructed within the agricultural ceremonies named mitote. After analyzing the ritual cargos used to personify the deities and the ties between them, this study will approach other elements normally led aside but that seem to be essential: objects and chants. Through the actions and roles played by the deities' personifications we will try to define the complexity of their presence along the ritual and the effect that it has on the executants of the mitote. [Key words : ritual, Cora (Náyeri), ritual cargo, maize, deer, agriculture, hunting, deities, identity, Nayarit.]

El venado cazador y el maíz agricultor. La identidad de las deidades en los mitote náyeri. Este artículo muestra cómo se constituye la identidad de las deidades náyeri à través de las ceremonias agrícolas llamadas mitote. Luego de analizar los cargos rituales destinados a personificar a las deidades y de los lazos que se establecen entre ellas, este trabajo aborda otros elementos que, aunque descartados comúnmente de la reflexión sobre las representaciones de las deidades, parecen ser también componentes esenciales: los objetos y los cantos. A partir de las acciones y de los roles asumidos por las distintas personificaciones de las deidades, se intentará definir la complejidad de su presencia en el ritual y los efectos que genera en los ejecutantes del mitote. [Palabras claves : ritual, coras (náyeri), cargos rituales, maiz, venado, agricultura, caza, deidades, identidad, Nayarit.]

* Doctorante, Université de Paris X-Nanterre/Laboratoire d'ethnologie et sociologie comparative, Maison René-Ginouvès (Archéologie et ethnologie), 21 allée de l'université, 92023 Nanterre cedex [margarita_valdovinos@hotmail.com].

Journal de la Société des Américanistes, 2008, 94-1, pp. 231-250. (O Société des Américanistes. 


\section{INTRODUCTION}

Chez les Náyeri ou Cora de l'ouest du Mexique ${ }^{1}$, la chasse est une activité fortement valorisée même si la proie par excellence, le cerf à queue blanche (Odocoileus virginianus), se fait de plus en plus rare. En tout cas, la mythologie comme le rituel témoigne de l'importance accordée à cette activité et au cervidé. La manifestation la plus frappante de celle-là en est le rituel nommé mitote ${ }^{2}$, dont la célébration recommande la chasse au cerf et où l'action rituelle la met en scène. Dans ce contexte, la chasse et le cerf sont traités en parallèle à l'agriculture et au maïs ${ }^{3}$. Ce parallélisme invite l'ethnologue à analyser en même temps l'utilisation de ces deux éléments (cerf et maïs) et le processus de leur obtention dans la construction de l'identité des divinités qui leur sont associées.

Dans les pages qui suivent, il s'agira de considérer les divinités comme la superposition de diverses présences qui apparaissent simultanément dans les actions rituelles. En effet, leur identité, telle qu'elle se construit au cours du mitote, résulte de l'interaction de leurs différentes composantes. Si les images du cerf et du maïs contribuent à marquer l'identité complexe des divinités, les processus (la chasse et l'agriculture) par lesquels les Náyeri les obtiennent le sont également. En conséquence, les figures des agents humains, qui apparaissent comme les acteurs de la chasse, de l'agriculture et des processus culinaires, interviennent aussi dans la construction identitaire des divinités.

Afin d'analyser de quelle manière les Náyeri donnent à voir leurs divinités au cours d'un mitote, il est indispensable d'accorder une attention particulière à l'utilisation des charges rituelles dont le but explicite est de personnifier les entités qui apparaissent comme composants des divinités. Toutefois, il convient d'accorder une attention égale au rôle joué par d'autres éléments dans la représentation des divinités : les objets rituels et les chants.

\section{LES MITOTE NÁYERI}

Les mitote sont des rituels qui ont pour objet privilégié la culture du maïs. Ils ont lieu trois fois par an en coïncidence avec les moments importants du cycle agricole : les semailles (juin), la récolte (octobre) et le stockage des grains (janvier). Ces rituels sont célébrés par chaque communauté ${ }^{4}$, mais également par certains groupes cognatiques qui ont hérité cette tradition de leurs ancêtres ${ }^{5}$. Chaque mitote dure entre trois et cinq jours pendant lesquels ont lieu un certain nombre de préparatifs : aménagement de l'espace rituel, élaboration du repas qui sera consommé et obtention d'objets considérés comme indispensables pour l'exécution du rituel. Les activités des participants se concentrent progressivement sur le terrain désigné pour le rituel, jusqu'à la veille du dernier jour, moment à partir duquel plus personne ne quitte l'endroit jusqu'à la fin du mitote. 
Après le coucher de soleil de la dernière nuit, le chanteur prend place face à l'autel, situé toujours à l'est de l'espace rituel. Il installe son arc musical (tunámï) devant lui et se met à jouer et à chanter. Les paroles de son chant incitent les participants à danser autour du feu, placé au centre du terrain, en suivant le sens contraire des aiguilles d'une montre. Les chants et la danse sont exécutés durant toute la nuit et ne s'arrêtent qu'au moment où le soleil atteint le zénith. Cette pause permet aux participants de partager un repas. Les chants et la danse reprennent ensuite jusqu'au soir ${ }^{6}$.

Pendant cette phase, les participants exécutent des pas de danse et des gestes qui permettent d'identifier les thèmes développés par le chanteur ${ }^{7}$. La relation qu'entretient la célébration des mitote avec le calendrier agricole conduit à privilégier les références à la culture du maïs. Toutefois, le thème de la chasse au cerf y apparaît également ${ }^{8}$. Chaque chant sert de guide pour les participants du rituel qui, selon leurs charges, sont invités à manipuler des objets et à les déplacer à travers le circuit de la danse.

La mise en place de chaque mitote requiert un minimum de participants. Ce groupe, qui comprend entre huit et quinze personnes, correspond aux détenteurs des charges rituelles : le maître de cérémonie et le responsable de l'espace rituel (principales), le chanteur et un groupe de jeunes individus qui assument des charges rituelles liées à la personnification des divinités. Ce dernier groupe se rassemble autour de deux personnages centraux correspondant à deux divinités : Tahá, « Notre Frère Aîné », et Tatyí, « Notre Mère » 9 .

\section{LA PERSONNIFICATION DES DIVINITÉS}

Dans le mitote, les jeunes détenteurs de charges apparaissent comme des personnifications de certaines divinités náyeri souvent présentes dans les récits mythologiques. Leurs charges, dont ils sont responsables pendant cinq ans, sont réparties dans deux sous-groupes, l'un féminin et l'autre masculin. À l'intérieur de chacun de ces sous-groupes, il existe trois échelons que les détenteurs occupent successivement, de sorte que tout participant reste attaché à une responsabilité rituelle pendant quinze ans (Figure 1) ${ }^{10}$.

\begin{tabular}{|l|l|l|l|}
\hline \multicolumn{1}{|c|}{ Dénominatif } & Âge des participants & Participants masculins & Participants féminins \\
\hline « enfants » & 5 à 10 ans & Ha'atsikan & Teih \\
\hline « auxiliaires » & 10 à 15 ans & Ha'atsikan tï baïre & Teih tï baïre \\
\hline « jeunes » & 15 à 20 ans & Narï & Kúhkamua \\
\hline
\end{tabular}

FIG. 1 - Les charges rituelles du mitote. 


\section{Les « enfants » du mitote}

Le premier échelon de charges est assumé par deux enfants d'environ cinq ans. Un garçon devient Ha'atsikan, identifié à Tahá, " Notre Frère Aîné », et lié au cerf. De son côté, une fillette assume le rôle de Teih, synonyme de Tatyí, « Notre Mère ", divinité proche du maïs. Ils sont choisis parmi les enfants conformément aux rêves du principal. Durant les cinq ans que dure leur charge, les autres participants du rituel utilisent Ha'atsikan et Teih comme des termes de référence et d'adressent à ces enfants, surtout au cours des conversations concernant la célébration des mitote.

Une fois qu'ils reçoivent leurs charges, ces enfants doivent se présenter à tout mitote, ainsi qu'à d'autres cérémonies du cycle rituel célébrées au cours des cinq années suivantes. Pendant leur participation, ils sont habillés la plupart du temps avec les éléments attribués à chacune des divinités qu'ils sont censés personnifier. Le garçon porte sur chaque côté de la tête des bâtons à plumes en forme de bois de cerf, sur les tibias des hochets produisant un bruit semblable à « celui que font les cerfs dans la forêt ", un bandeau sur le front qui protège une couronne de plumes de perroquet et des lanières qu'il porte en bandoulière, généralement accompagnées d'un carquois. La fillette arbore des vêtements blancs et des colliers fabriqués avec des grains variés. Elle porte sur la tête un foulard blanc recouvert de rosettes de rubans de différentes couleurs " comme les fleurs qui décorent le maïs » (Figures 2 et 3).

\section{Les « auxiliaires 》}

Les charges d'« auxiliaires 》 sont attribuées à ceux qui ont accompli les charges d'《 enfants » quand ils atteignent une dizaine d'années ${ }^{11}$. Ces charges exigent une connaissance plus pointue des actions rituelles car leur rôle est de guider les déplacements des « enfants » dans l'espace rituel et de les remplacer dans les actions compliquées ou rapides, surtout quand elles impliquent la manipulation d'objets. Cette expérience est acquise par leurs porteurs justement grâce à leur participation antérieure en tant qu' « enfants ».

Les « auxiliaires » ne portent pas de costume spécifique. Toutefois, l'étroite relation qu'ils entretiennent avec les " enfants» au cours des actions rituelles témoigne de leur proximité. En effet, « auxiliaires » et « enfants » de chaque sexe apparaissent toujours côte à côte, ce qui conduit à les percevoir comme des personnages dédoublés. La fusion de leurs identités est mise en valeur dans les chants, où leurs noms sont souvent utilisés comme des synonymes. 


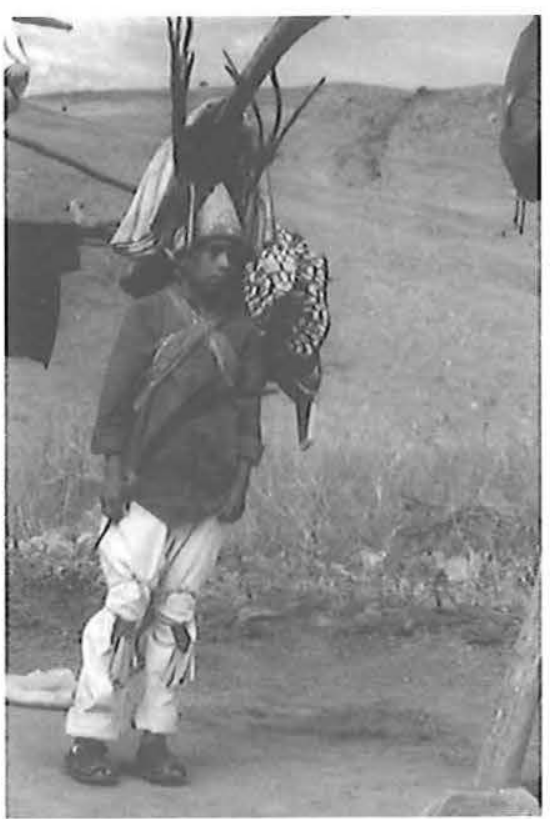

FIG. 2 - Ha'atiskan regarde les danses (cliché Valdovinos).

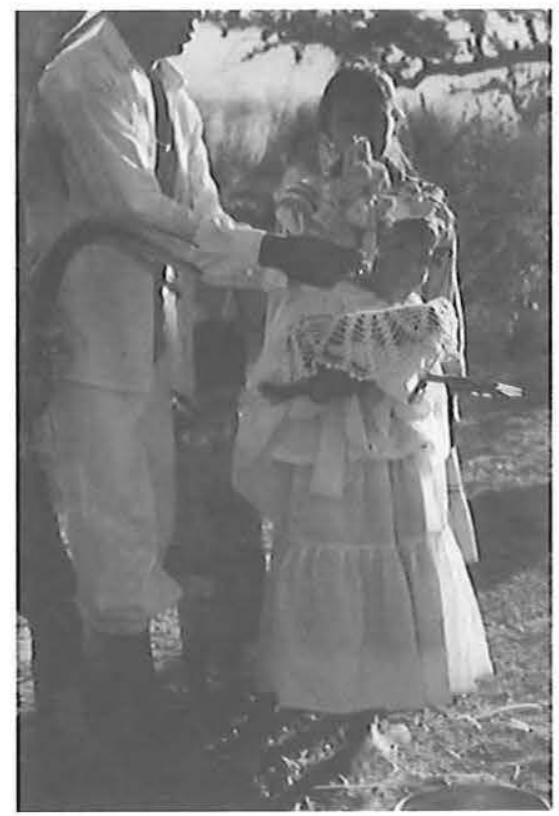

Fıg. 3 - Teih reçoit le maïs (cliché Valdovinos).

\section{Les « jeunes 》 du mitote}

Ces charges sont assumées par un couple d'enfants, une fois accomplies les charges d'« auxiliaires », c'est-à-dire vers l'âge de quinze ans. Les " jeunes », ayant acquis une connaissance approfondie de l'action rituelle grâce à leur participation en tant qu' « enfants », puis comme " auxiliaires », accèdent à un nouveau statut leur permettant de se déplacer plus librement dans l'espace rituel et de prendre en charge d'autres actions. Cette fois, la jeune fille représente Kúhkamua, qui entretient le feu de la cuisine rituelle et assume la préparation du maïs. De son côté, le jeune homme représente Narï, le gardien du feu central qui s'occupe aussi de la préparation culinaire du cerf chassé.

Les personnages joués par les « jeunes » possèdent certains attributs vestimentaires mais, à la différence des « enfants », ils ne les utilisent que durant des moments particuliers du rituel. Quand la situation l'exige, Narï porte des bâtons à prières derrière les oreilles imitant les bois d'un cerf, une couronne de plumes de perroquet et des hochets attachés aux jambes; il tient entre ses mains, placées derrière son dos, un bâton décoré avec la queue d'un cerf à queue blanche. De son côté, quand Kúhkamua est invitée à manipuler le maïs, elle porte une couronne de fleurs et un bâton à prières derrière la tête. Elle tient alors dans chaque main un petit bouquet de fleurs qu'elle agite durant sa danse. 
Dans le cas des « enfants » et dans celui des « jeunes », la personnification des divinités est en grande partie exprimée par l'utilisation de costumes rituels et l'attribution de noms propres. Les « auxiliaires » se situent sur une sorte d'échelon de passage entre la charge des «enfants », avec laquelle ils s'identifient par leur nom, et la charge des « jeunes », dont les détenteurs peuvent, comme eux, manipuler certains objets rituels et traverser l'espace afin d'accomplir les tâches qui leur sont désignées. La position transitoire des charges d'« auxiliaires » manifeste un principe qui semble constitutif de l'identité des divinités náyeri, combinant des éléments appartenant à différentes composantes, en l'occurrence l'identité des « enfants » et le dynamisme des « jeunes ».

\section{LA MISE EN SCÈNE DES DIVINITÉS}

Dans l'action rituelle, les fonctions des porteurs de charges de chaque niveau (« enfants », « auxiliaires », « jeunes ») répondent à des principes d'ordre différent, tout en étant complémentaires. Cette complémentarité invite à analyser en détail le mode d'action lié à chacune des charges.

Avant de continuer, il convient de revenir sur un point. En plus des personnifications assumées par les porteurs des charges, il est possible d'observer au cours de l'action rituelle une autre manifestation de la présence des divinités, par l’intermédiaire des éléments alimentaires qui leur sont étroitement liés : tandis qu'un cerf spécialement chassé pour l'occasion apparaît comme un composant de Tahá, une botte d'épis de maïs est considérée comme étant partie de Tatyí.

La présence de ces éléments ne doit pas simplement renvoyer à l'image des divinités auxquelles ils sont liés. Ils doivent apparaître comme de véritables agents capables d'entretenir des relations avec les humains ; ces relations étant définies comme relevant de la " chasse » et de l'« agriculture » ${ }^{12}$. Dans le contexte rituel, tandis que la chasse est considérée comme une activité masculine, tout ce qui concerne le maïs est envisagé comme la tâche des femmes. Ainsi, dans les premiers jours du rituel, les hommes se consacrent à la recherche du cerf et les femmes à la décoration des épis de maïs.

Quelques jours avant le mitote, les chasseurs observent une période de jeûne et de prières afin de préparer une partie de chasse aux cerfs. Une période de purification est considérée comme une condition pour la réussite de l'expédition. Sa fonction présumée est de pousser le cerf à s'approcher du chasseur. Lors de la capture d'un cerf, le chasseur doit ramener sa proie jusqu'à la maison et s'occuper du traitement de la chair, du cuir et des bois ${ }^{13}$.

Dans le cas du maïs, on prévoit trois ou quatre épis qui, attachés entre eux, sont décorés par les femmes de petites fleurs ${ }^{14}$. Le maïs rituel et les fleurs destinées à son ornement sont cultivés au centre du champ (esp. milpa) où, à la différence des grains jetés dans des sillons parallèles ordinaires, ils sont semés suivant un mouvement centrifuge. 
Le couple d'« enfants » se rend à l'endroit où le mitote va être célébré dès le premier jour des préparatifs, leur présence étant requise pendant toutes les cérémonies oratoires célébrées à ce stade. Les « enfants » doivent aussi participer à toutes les danses nocturnes, où ils sont souvent au centre des actions décrites par les chants. Leur responsabilité principale consiste à tenir, à des moments déterminés, des objets qui leur sont attribués : la fillette porte une calebasse contenant la botte d'épis de maïs et le garçon une gourde remplie d'eau servant à asperger les participants lors des prières ${ }^{15}$.

Les objets rituels portés par les " enfants ", ainsi que les attributs vestimentaires de ces derniers, contribuent à établir un parallélisme entre eux, d'un côté, et le cerf et le maïs, de l'autre. Cette identification leur permet d'assumer la personnification des divinités Tahá et Tatyí ${ }^{16}$. Toutefois, la limitation des actions attribuées aux « enfants » ne permet qu'une personnification de caractère passif : ceux-ci ne font que tenir les objets rituels qui soulignent simplement la nature de l'entité qu'ils personnifient.

Les « auxiliaires » ont pour fonction d'attribuer du mouvement aux actions des « enfants ». Ils doivent les guider dans leurs déplacements et manipuler leurs objets rituels autour de l'espace rituel. Par leurs actions, les « auxiliaires » insèrent les « enfants » dans une dynamique d'interaction réelle leur permettant de surmonter leur personnification passive en les conduisant à se déplacer entre les autres participants du mitote. Ces actions ont comme résultat la construction d'un espace où tous les exécutants, les " enfants » et leurs objets rituels inclus, deviennent des participants (Figure 4).

Finalement, les "jeunes » ajoutent une dimension temporelle aux actions rituelles en assurant la fonction de transformateurs du cerf et du maïs présents dans le mitote. En tant que Narï et Kúhkamua, les « jeunes » permettent la mise en scène des processus d'obtention du maïs (agriculture) et du cerf (chasse) et de leur traitement culinaire. Afin de comprendre l'importance de la fonction des " jeunes ", il faut cependant considérer que ce n'est que grâce à la personnification des «enfants », menés à l'interaction par leurs " auxiliaires », que les " jeunes » peuvent accomplir cette transformation (Figure 5).

La configuration résultante permet de souligner un aspect qui était resté imperceptible jusqu'ici. Tandis que, dans les premiers moments du rituel, les composantes des divinités (objets votifs et " enfants ») assument des fonctions dont l'intention est d'accentuer l'identification entre les différents éléments qui sont à l'origine de leur identité (humains et cerf/maïs), les composants des divinités qui apparaissent par la suite («auxiliaires» et «jeunes») tendent à marquer une différenciation entre ces éléments-là.

Les « auxiliaires » insistent pour séparer matériellement les « enfants » des objets rituels qu'ils portent indiquant ainsi que les uns sont dynamiques et les autres inertes. À cette fin, d'un côté, les " auxiliaires » poussent les « enfants » à se déplacer dans l'espace rituel et, d'un autre, ils assument eux-mêmes les 


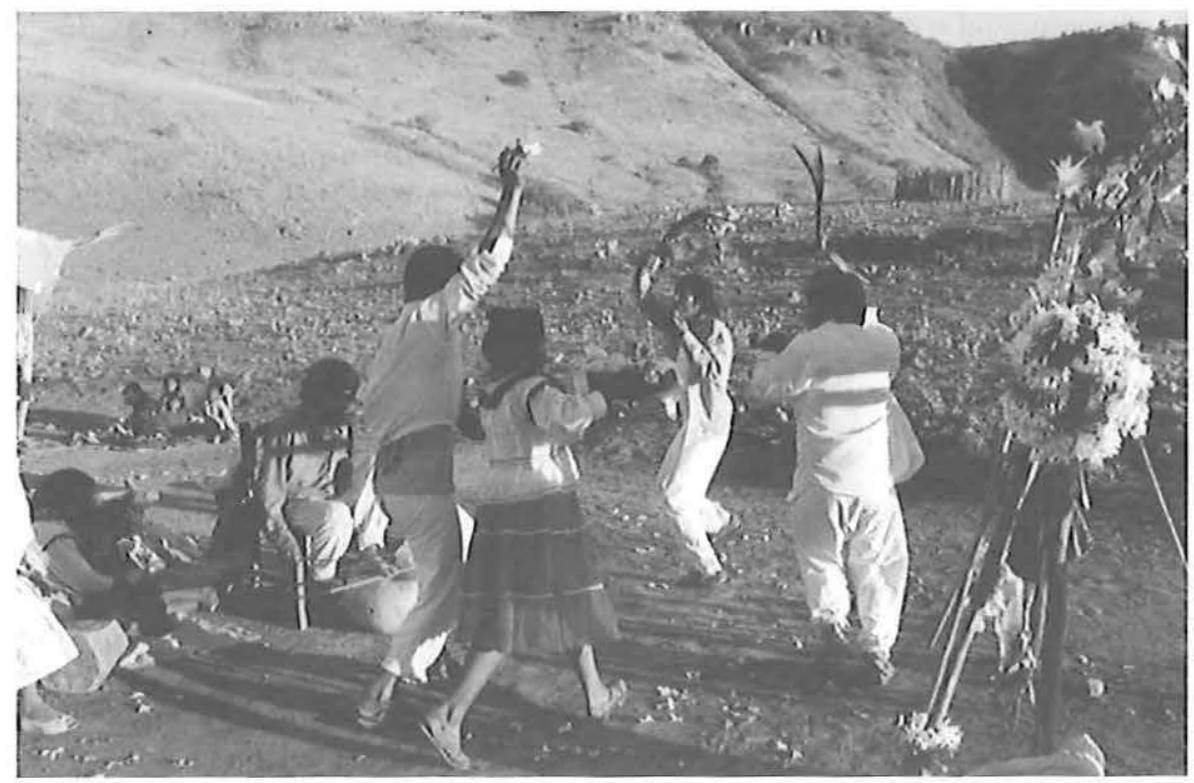

Fig. 4 - Les « auxiliaires » déplacent en dansant les objets votifs des « enfants » (cliché Valdovinos).

\begin{tabular}{|l|l|l|l|}
\hline $\begin{array}{c}\text { Représentations } \\
\text { des divinités }\end{array}$ & Détenteurs masculins & \multicolumn{1}{c|}{ Détenteurs féminins } & \multicolumn{1}{c|}{ Fonetion } \\
\hline objets rituels & gourde d'eau & $\begin{array}{l}\text { calebasse avec épis } \\
\text { de maïs }\end{array}$ & $\begin{array}{l}\text { identification avec } \\
\text { produits alimentaires }\end{array}$ \\
\hline « enfants » & Ha'atsikan & Teih & personnification \\
\hline « auxiliaires » & Ha'atsikan tï baïre & Teih tï baïre & interaction \\
\hline « jeunes » & Narï & Kúhkamua & transformation \\
\hline
\end{tabular}

FIG. 5 - Les modes d'action des détenteurs des charges.

déplacements des objets votifs considérés comme étant inertes. La participation des « jeunes » tend également à souligner une différenciation entre les « enfants » et les « objets votifs ", mais cette fois pour identifier les uns comme proies, grains et produits alimentaires, et les autres comme agriculteurs, chasseurs et consommateurs (Figure 6).

Or la contradiction entre ces deux tendances opposées fait émerger un nouvel enjeu qui exige une solution dans l'action rituelle : comment envisager des divinités dont les composants montrent simultanément des tendances à l'identification et à la différenciation? 


\begin{tabular}{|c|c|c|}
\hline Composantes & Processus & Tendance \\
\hline cerf/maïs & $\begin{array}{l}\text { Ils apparaissent comme dotés d'une intentionna- } \\
\text { lité à l'instar des humains }\end{array}$ & \multirow{2}{*}{ identification } \\
\hline Ha'atsikan/Teih & $\begin{array}{l}\text { Ils personnifient des attributs appartenant au cerf } \\
\text { et au maïs }\end{array}$ & \\
\hline $\begin{array}{l}\text { Ha'atsikan tï baïrel } \\
\text { Teih tï baïre }\end{array}$ & $\begin{array}{l}\text { Ils manipulent les objets votifs des « enfants » et } \\
\text { guident séparément ces derniers }\end{array}$ & \multirow{2}{*}{ différenciation } \\
\hline Narï/Kúhkamua & $\begin{array}{l}\text { Ils mettent en scène la transformation des objets } \\
\text { rituels en nourriture }\end{array}$ & \\
\hline
\end{tabular}

FIG. 6 - Identification et différenciation entre les composantes des divinités.

\section{LES DIVINITÉS INVISIBLES}

Les chants émis pendant la nuit du mitote offrent des éléments qui proposent aux participants une nouvelle « apparence » des divinités, caractérisée alors par leur invisibilité. C'est justement cette nouvelle image sonore des divinités qui offre une solution à la contradiction entre l'identification et la différenciation des composantes examinées précédemment. Cette fois, les divinités qui émergent par les chants ne sont pas considérées par les exécutants du rituel comme de simples évocations. Elles sont conçues - en tout cas par les spécialistes rituels - comme authentiquement présentes dans l'espace rituel ${ }^{17}$.

Pour faire « apparaître » les divinités, le chanteur est censé utiliser toute sa dextérité afin de décrire à travers ses paroles tout ce qui entoure l'arrivée de ces entités invisibles dans l'espace rituel. Pour y parvenir, il se sert des descriptions détaillées qui narrent le voyage des divinités depuis les endroits lointains d'où elles sont parties jusqu'à leur rencontre hic et nunc avec ceux qui célèbrent le mitote. Ainsi, c'est à travers la voix et la narration du chanteur que ces divinités se font présentes auprès des participants.

Le premier couple de divinités qui surgit par les chants correspond à des doubles des « enfants » qui, à la différence de ces derniers, sont invisibles tout au long du rituel. Ces « enfants invisibles » sont appelés aussi Ha'atsikan et Teih et, comme l'indiquent les paroles des chants, ils partagent avec eux les mêmes attributs vestimentaires. En fait, c'est par un jeu entre la concordance et la discordance des actions de ces deux couples d'enfants qu'il est possible soit de les confondre, ce qui arrive aux néophytes, soit de les distinguer, comme le font les spécialistes rituels.

Un exemple de la distinction entre les « enfants » et les " enfants invisibles » est donné au moment où les derniers arrivent dans l'espace rituel. En décrivant en détail l'habillement de ces personnages dans son chant, le chanteur construit leur présence. Cependant, il doit également montrer que, au-delà des apparences, il n'est pas en train de décrire les caractéristiques vestimentaires des « enfants » qui 
se trouvent déjà dans l'espace rituel. Pour cela, il s'efforce de donner une existence concrète aux " enfants invisibles » tout en montrant emphatiquement que la présence des « enfants » est également prise en compte dans son chant. À cet effet, il souligne que les «enfants » sont déjà en train d'exécuter certaines actions différentes de celles qu'il attribue aux " enfants invisibles " ${ }^{18}$.

Un deuxième couple de divinités qui apparaît dans le chant correspond à Húrimua, "La Donatrice de la Vie », et à Túramuna, «le Grand Cerf de la Forêt ». Ces autres composants des divinités sont aussi de nature invisible, mais à la différence de celles qui ont été présentées plus haut, aucune description de leur personne n'est utilisée pour les donner à voir. Bien au contraire, les paroles des chants évoquent leur présence à travers l'énumération de caractéristiques autres que leur apparence physique : la description des lieux où les divinitiés sont censées habiter et depuis lesquels elles contemplent le rituel, la nature de leur puissance, les faveurs qu'elles font aux humains et l'immortalité dont elles jouissent.

En contraste avec les " enfants invisibles », ces autres divinités chantées, bien que considérées aussi comme authentiques, apparaissent personnifiées dans le rituel par deux des détenteurs des charges : le jeune Narï et la fillette Teih. On reviendra sur ce point plus loin. Pour l'instant ce qui doit être souligné est le changement de perspective que ces divinités invisibles apportent par leur présence. En étant invisibles, elles arrivent à déplacer la contradiction posée par l'identification et la différentiation vers un terrain où l'enjeu ne réside plus dans l'apparence externe ou dans la capacité d'action ${ }^{19}$, mais dans l'assignation de rôles à travers lesquels chacune des composantes des divinités se fait présente au cours de l'action rituelle.

\section{L'ASSIGNATION DE RÔLES RITUELS}

Maintenant que toutes les composantes des divinités ont été présentées, il devient possible d'aborder un dernier aspect : l'assignation de rôles permettant la mise en scène de la chasse, de l'agriculture et du traitement culinaire réservé au cerf et au maïs. À cet effet, l'analyse des actions accompagnant l'énonciation de deux extraits de chants est pertinente ${ }^{20}$. Il s'agit de chants concernant justement la chasse au cerf et le traitement agricole et culinaire accordé au maïs. Dans les deux cas, la séquence des actions décrites dans ces chants est traduite en actes et gestes par les exécutants du rituel.

Dans le premier cas, le chant du Túramuna, " le Grand Cerf de la Forêt », contient une description détaillée de la chasse au cerf. Le chant expose comment Ha'atsikan, précédant un groupe de chasseurs, part à la chasse du cerf Túramuna dont le rôle est interprété par le jeune Narï. Suivant les indications données par Ha'atsikan, les chasseurs préparent des lassos et partent à la recherche du grand cervidé. L'un d'entre eux cherche les traces laissées par le cerf dans la forêt, 
pendant que le reste des chasseurs pose des pièges en forme de toiles d'araignée faites avec les lassos. Ils parviennent à l'encercler plusieurs fois, mais Túramuna réussit toujours à s'évader. Ha'atsikan décide alors de le chasser lui-même et prépare son arc et ses flèches. Il poursuit Túramuna jusqu’à ce qu'il le cerne et le tue d'une flèche. Il demande alors aux chasseurs de le ramener avec eux. Quand Tatyí, " Notre Mère », se rend compte de ce qui s'est passé, elle va sur place et informe Ha'atsikan qu'il vient de tuer son propre frère. Elle pleure et décide de lui rendre la vie avant de s'en aller. Ha'atsikan demande quand même aux chasseurs d'écorcher le corps et de le déposer dans un trou recouvert de braises ${ }^{21}$. Une fois que le cerf est entièrement recouvert de branches, il revit et se met à danser.

Le deuxième extrait correspond au chant du maïs. Ici, Ha'atsikan demande à Teih de rendre le maïs pour qu'il soit égrené, puis grillé ou bouilli ${ }^{22}$ par Kúhkamua. Teih se montre angoissée par la perte de son " enfant ». Elle demande alors aux esprits des ancêtres (tyakuatye) d'essayer de le sauver. Les esprits répondent que cette situation a été prévue depuis longtemps et qu'elle est inévitable. Teih souffre de la mort imminente de son « enfant » qui correspond à la botte d'épis de maïs ${ }^{23}$. Finalement elle accepte de rendre à Kúhkamua son « enfant » et cette dernière le cuisine. Les esprits des ancêtres dévoilent alors à Teih que son « enfant » ne va pas mourir par la main de Kúhkamua. Cette dernière continue sa préparation culinaire (Figures 7 et 8 ).

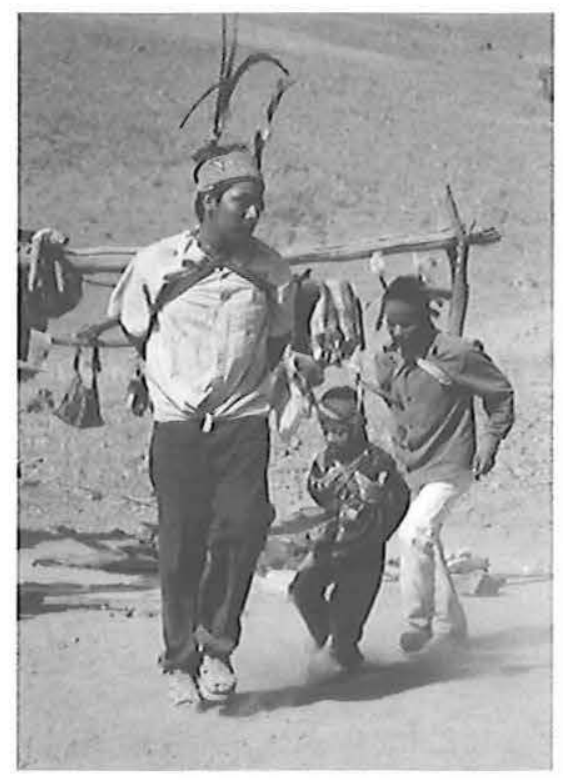

Fig. 7-Narï joue le cerf et échappe aux chasseurs-cerfs qui le poursuivent (cliché Valdovinos).

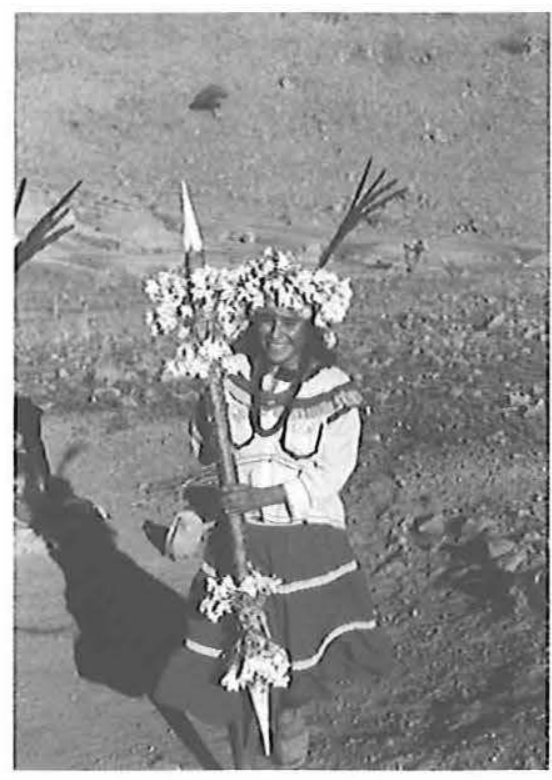

FIG. 8-Kúhkamua s'apprête à mettre en scène les semailles à l'aide d'un bâton à fouir (coa) (cliché Valdovinos). 
Les deux extraits des chants présentent une structure similaire dont l'aboutissement est la transformation du cerf ou du maïs en nourriture (Figure 9). Par l'intervention d'un intermédiaire (a), apparaît d'abord une situation de transformation mal réussie (b), puis l'accomplissement du processus de transformation (c), et enfin a lieu le dévoilement d'un secret qui contredit les apparences (d), c'est-à-dire qui réaffirme que la destruction entraînée par ricochet dans le processus de transformation du cerf et du maïs n'implique pas la mort de l'entité qui en était la victime. Finalement, un nouveau médiateur conclut la transformation du cerf et du maïs en nourriture (e).

\begin{tabular}{|l|l|l|}
\hline \multicolumn{1}{|c|}{ ActionlEntité } & \multicolumn{1}{c|}{ Cerf } & \multicolumn{1}{c|}{ Maïs } \\
\hline $\begin{array}{l}\text { a) appel à un } \\
\text { intermédiaire }\end{array}$ & $\begin{array}{l}\text { Ha'atsikan demande aux } \\
\text { hariwame de tuer Túramuna } \\
\text { «le Grand Cerf » }\end{array}$ & $\begin{array}{l}\text { Ha'atsikan demande à Teih de } \\
\text { rendre le maïs à Kúhkamua }\end{array}$ \\
\hline $\begin{array}{l}\text { b) empêchement de } \\
\text { l'intermédiaire }\end{array}$ & $\begin{array}{l}\text { les hariwame n'arrivent pas à tuer } \\
\text { Túramuna }\end{array}$ & $\begin{array}{l}\text { Teih essaie de sauver le maïs en } \\
\text { parlant aux tyahkuatye }\end{array}$ \\
\hline $\begin{array}{l}\text { c) confrontation entre } \\
\text { dédoublements }\end{array}$ & $\begin{array}{l}\text { Ha'atsikan tue le cerf } \\
\text { (représenté par Narï) }\end{array}$ & $\begin{array}{l}\text { Teih donne le maïs à Kúhkamua } \\
\text { et cette dernière le fait cuire }\end{array}$ \\
\hline $\begin{array}{l}\text { d) révélation de l'iden- } \\
\text { tité de la victime }\end{array}$ & $\begin{array}{l}\text { Tatyí dit à Ha'atsikan que le cerf } \\
\text { est son frère et lui rend la vie }\end{array}$ & $\begin{array}{l}\text { les esprits renseignent Teih sur } \\
\text { l'immortalité de son " enfant » }\end{array}$ \\
\hline $\begin{array}{l}\text { e) transformation en } \\
\text { nourriture }\end{array}$ & $\begin{array}{l}\text { Ha'atsikan demande aux } \\
\text { hariwame de cuisiner le cerf }\end{array}$ & $\begin{array}{l}\text { Kúhkamua cuisine l'« enfant » de } \\
\text { Teih, qui est le maïs. }\end{array}$ \\
\hline
\end{tabular}

FIG. 9 - Les processus de transformation du cerf et du maïs dans les chants rituels.

Au cours du mitote, les détenteurs des charges rituelles mettent en scène les interactions qu'entretiennent les Náyeri avec les deux produits alimentaires qui apparaissent au centre de l'action. D'un côté, Ha'atsikan et Narï, par l'intermédiaire de Ha'atsikan tïbaïre, assurent la mise en scène des processus concernant le cerf. D'un autre côté, Teih et Kúhkamua, à l'aide de Teih tï baïre, montrent le traitement qui est fait du maïs. La distribution des rôles utilisée dans le mitote accorde à Ha'atsikan (« enfant ») la position du chasseur et celle de cerf à Narï ( « jeune »). De leur côté, Kúhkamua (« jeune ») assume le rôle d'agriculteur et la fillette Teih celle de la procréatrice du maïs cultivé (Figure 10) ${ }^{24}$.

\begin{tabular}{|c|c|c|c|}
\hline Processus & Dédoublement & Segmentation & Processus \\
\hline \multirow{2}{*}{ chasse au cerf } & $\begin{array}{l}\text { Ha'atsikan } \\
\text { « enfant » }\end{array}$ & $\begin{array}{l}=\text { chasseur } \\
\neq \text { cerf }\end{array}$ & \multirow{2}{*}{$\begin{array}{l}\text { Ha'atsikan (chasseur) tue le cerf } \\
\text { (Narï) }\end{array}$} \\
\hline & $\begin{array}{l}\text { Narï } \\
\text { « jeune» }\end{array}$ & $\begin{array}{l}\neq \text { chasseur } \\
=\text { cerf }\end{array}$ & \\
\hline \multirow{2}{*}{ culture du maïs } & $\begin{array}{l}\text { Teih } \\
\text { « enfant » }\end{array}$ & $\begin{array}{l}\neq \text { agriculteur } \\
=\text { maïs }\end{array}$ & \multirow{2}{*}{$\begin{array}{l}\text { Kúhkamua (agriculteur/cuisi- } \\
\text { nière) s'occupe du maïs (Teih) }\end{array}$} \\
\hline & $\begin{array}{l}\text { Kúhkamua } \\
\text { " jeune » }\end{array}$ & $\begin{array}{l}=\text { agriculteur } \\
\neq \text { maïs }\end{array}$ & \\
\hline
\end{tabular}

FIG. 10 - Les rôles assumés par les divinités dans l'action rituelle. 
La mise en scène des deux extraits de chants cités signale l'intervention des divinités - à travers les personnifications assumées par les détenteurs des charges - en tant que médiatrices entre les humains et les produits qu'elles manipulent jusqu'à l'obtention de nourriture par les premiers. La position des divinités est cependant loin d'être simple. Leur médiation consiste à assumer simultanément l'ensemble des rôles exigés pour donner à voir la relation entre hommes et produits alimentaires, c'est-à-dire que les divinités apparaissent comme étant en même temps le terme qui souffre de la transformation (cerf et maïs) et celui qui exécute la transformation (chasseur, agriculteur et cuisinier).

\section{LA REDÉFINITION DU RÔLE DES HUMAINS}

En occupant l'ensemble des rôles dans la mise en scène de la transformation du cerf et du maïs en nourriture, les différentes composantes divines amènent les humains à accomplir une autre transformation qui les concerne au premier chef et qui propose une solution à la conception qu'ils ont de l'approvisionnement des produits considérés comme archétypes de leur nourriture. À la différence de la transformation subie dans la vie réelle par le cerf et le maïs, celle-ci ne suppose pas un changement d'état. Elle implique, cependant, la modification de la manière dont les Náyeri conçoivent leur relation avec le cerf et le maïs.

Si l'on suit la progression de l'ensemble des actions exécutées dans le mitote, on voit que, dans un premier temps, pendant les préparatifs, les Náyeri apparaissent comme les participants actifs de la relation, définie comme étant de " chasse », qu'ils entretiennent avec le cerf, et de celle d' " agriculture », qu'ils entretiennent avec le maïs. Ainsi, les hommes partent à la chasse et les femmes s'occupent de l'élaboration culinaire des produits faits à base de maïs ${ }^{25}$. À ce stade, le cerf et le maïs se voient assigner une position passive, car ils se font chercher, cultiver et cuisiner respectivement.

Ensuite, au cours des danses guidées par les chants, ce sont les différentes composantes divines qui détiennent l'ensemble des termes de la relation que les humains entretiennent normalement avec le cerf et le maïs. Elles assument, d'un côté, les rôles de ceux qui cultivent, chassent et préparent le cerf et le maïs et, d'un autre côté, les rôles mêmes du cerf et du maïs. De ce jeu de rôles, les Náyeri retiennent le caractère actif du cerf et du maïs, qu'ils projettent ensuite dans la relation qu'ils entretiennent avec ces entités en dehors du rituel. Ainsi, la chasse au cerf peut être interprétée comme une relation où le cerf se rend lui-même aux chasseurs et le maïs se transforme lui-même en nourriture, ainsi qu'en rend compte la mythologie ${ }^{26}$.

Le repas cérémoniel qui marque la fin des danses apparaît ainsi comme l'acceptation de la part des humains des actions du cerf et du maïs. Par ce repas, les premiers projettent vers les seconds la responsabilité de l'obtention des 
produits alimentaires : ce sont le cerf et le maïs qui se livrent eux-mêmes aux hommes, tout en se préservant (Figure 11).

\begin{tabular}{|l|l|l|}
\hline \multicolumn{1}{|c|}{ Moment du rituel } & \multicolumn{1}{c|}{ Rôle actif } & \multicolumn{1}{c|}{ Rôle passif } \\
\hline préparatifs & humains & cerf/maïs \\
\hline chants et danses & divinités & divinités \\
\hline repas & cerf/maïs & $\bigcup_{\text {humains }}$ \\
\hline
\end{tabular}

FIG. 11 - Le changement de position du rôle des humains.

En tant que participant actif, le cerf peut être enfin considéré pleinement comme Tahá, " Notre Frère Aîné », puisque les Náyeri peuvent alors envisager dans cette relation que l'on peut qualifier de germanité non seulement des interactions de type compétition, où le résultat de la chasse dépend de l'habileté du chasseur, mais également des interactions de type complicité, où le cerf doit reconnaître les efforts de purification du chasseur et se rendre à lui par sympathie. Un résultat similaire concerne le maïs qui est appelé Tatyí, " Notre Mère ». Cette céréale n'est plus simplement considérée à travers une relation de type agricole où les Náyeri doivent s'occuper d'elle, mais plutôt dans une relation de type maternel où les Náyeri seraient les enfants et le maïs la mère, ils seraient dépendants de la céréale ${ }^{27}$.

Si ce retournement de positions entre les Náyeri, d'un côté, et le cerf et le maïs, de l'autre, permet aux premiers de concevoir les relations qu'ils entretiennent avec les seconds comme des relations de parenté, il en est de même pour les liens qu'ils ont avec les divinités, occupant des positions identiques à celles qui sont attribuées dans le mitote au cerf et au maïs. De ce fait, il est évident que toute relation qui est donnée à voir au cours du mitote s'inspire d'abord de relations entretenues entre humains et que celles-ci se projettent ensuite vers celles qu'entretiennent les humains avec des entités différentes.

\section{CONCLUSION}

Les divinités apparaissent comme les participants idéaux du mitote car, à travers leurs composantes, elles sont prêtes à assumer tous les rôles nécessaires pour l'accomplissement de la transformation du cerf et du maïs en nourriture. En même temps, leur mise en scène offre aux Náyeri une façon de transformer la vision qu'ils se font des relations qu'ils entretiennent avec ces autres entités du monde.

Les observations sur le mitote ici présentées montrent que la mise en scène des divinités náyeri contribue à exprimer la complexité de ces entités. Ainsi, en plus 
des objets rituels, trois charges rituelles (« enfants, « auxiliaires » et « jeunes ») sont nécessaires afin de créer une configuration reflétant l'ensemble des composants des divinités. À travers ces composantes s'expriment simultanément deux tendances opposées : l'une qui tend à unifier les différentes parties et les propriétés des divinités, tandis que l'autre souligne leur discontinuité. Mais, en raison de l'apparition des personnifications invisibles des divinités suggérées par les chants, surgit une nouvelle perspective : la nature des divinités - avec ses identifications et ses différenciations - est déplacée en faveur d'une approche relationnelle où ce qui compte est plutôt la distribution des rôles requis pour la mise en scène de la chasse et de l'agriculture. À ce moment, les détenteurs des charges qui assument déjà la personnification des divinités s'engagent dans les rôles nécessaires pour accomplir la transformation du cerf et du maïs : chasseur ou cerf, pour les garçons, et agriculteur ou maïs, pour les filles.

Chacune des personnifications des divinités náyeri dégagées par l'action rituelle apparaît donc insérée dans des réseaux relationnels différents entre les participants, entre elles-mêmes et entre les rôles qu'elles assument dans la mise en scène. En ce sens, la configuration rituelle peut être décrite non seulement comme une condensation de relations contradictoires (Houseman et Severi 1994), mais également comme une condensation de personnages engagés simultanément dans des perspectives différentes.

Les données náyeri permettent également de revenir sur la théorie du sacrifice telle qu'elle a été avancée par Robertson Smith (1969) ${ }^{28}$ et reprise par Hubert et Mauss (1968a) à travers l'idée que « le sacrifice est un moyen pour le profane de communiquer avec le sacré par l'intermédiaire d'une victime » (ibid., 1968b, p. 16). Dans leur analyse, le sacrifice implique la mise en interaction de trois termes : la victime qui sert d'intermédiaire, le sacrifiant et le dieu qui préside le sacrifice. Si, dans l'analyse des auteurs, le sacrifice apparaît, avant tout, comme un moyen utilisé par les hommes pour communiquer avec les dieux par l'intermédiaire d'une victime commune, dans le cas náyeri et selon l'approche envisagée dans ces pages, ce sont plutôt les divinités qui apparaissent comme les entités communes aux exécutants du rituel et comme archétypes de leur nourriture. Ainsi, la mise à mort ritualisée du cerf et du maïs assumée par les détenteurs des charges semble, pour les Náyeri, un moyen pour s'approcher de ces aliments et pour créer avec eux des liens stables socialement reconnus ${ }^{29}$. Pour cela, les divinités, entités mobiles par excellence, apparaissent comme des médiateurs exemplaires. *

* Manuscrit reçu en janvier 2007 et accepté pour publication en septembre 2007. 


\begin{abstract}
NOTES
Une première version de cette analyse a été présentée dans le séminaire de recherche « Guerre, chasse et sacrifice en Mésoamérique " proposé par Grégory Pereira et Alain Breton en janvier 2006. Depuis, j’ai pu avancer dans mes réflexions sur le mitote náyeri. Je remercie, pour leurs commentaires, les membres de l'atelier d'écriture des doctorants d'ethnologie du Laboratoire d'ethnologie et de sociologie comparative (2006-2007) avec qui j'ai pu discuter de ce texte. Je remercie aussi Martin Hervouët pour ses commentaires et, de manière particulière, François Berthomé qui a relu différentes versions de
\end{abstract} ce travail.

1. Les Náyeri habitent dans l'Ouest du Mexique, dans la partie de la Sierra Madre occidentale correspondant à l'État du Nayarit. Il s'agit d'une population indienne d'environ 18000 personnes qui partagent une même langue (cora) de la famille yuto-aztèque et un même type d'organisation sociale et politique. Pour cet article, je pars des observations que j'ai recueillies dans la communauté de Jesús Maria, où je réalise des séjours de terrain depuis 1997. Je m’appuie également sur mon étude de la tradition rituelle d'un groupe cognatique habitant le sud du territoire mariteco. Les cycles rituels d'autres communautés náyeri ont été décrits par Coyle (2001) et Guzmán (2002).

2. Les mitote sont considérés comme les rituels agricoles des Náyeri, bien qu'ils traitent également d'autres aspects. Par contraste, d'autres cérémonies sont organisées sur des thèmes faisant aussi allusion au cycle agricole, comme le cycle de vie du Christ. La présence constante et simultanée des danses et des chants constitue la caractéristique principale des mitote. Le mot mitote provient du nahuatl, langue qui a été utilisée dans la région par les missionnaires catholiques comme lingua franca. Le terme " mitote » et son équivalent cora " mehtyínyieita' aka » font effectivement référence à la danse. Mitote a servi aux premiers évangélisateurs pour désigner un type particulier de rituel païen caractérisé justement par la présence de danses. Pour des questions de simplicité et en dépit des controverses (Alvarado 2004), j'utiliserai ici le terme mitote.

3. Les Náyeri observent notamment des similitudes entre le cycle de vie et de croissance du maïs et celui du cerf. En effet, dans la région montagneuse habitée par les Náyeri, il n'y a qu'une seule saison des pluies (de juin à octobre), il ne peut donc y avoir qu'un cycle de travail agricole par an (González Ramos 1972, pp. 19-20). Au cours de ce cycle unique, le sarclage du terrain agricole coïncide avec le moment où les cerfs perdent leurs bois (avril-mai), tandis que l'éclosion des épis du maïs arrive en même temps que la poussée de leurs nouveaux bois (septembre-octobre).

4. Dans la région du Gran Nayar, une communauté comprend une population habitant un territoire délimité géographiquement dont les membres partagent des pratiques culturelles, une variante dialectale de la langue cora et une organisation politique établie autour d'un gouvernement traditionnel, c'est-à-dire selon des règles internes déterminées par les anciens de chaque groupe.

5. Les groupes cognatiques náyeri s'organisent autour d'un homme considéré comme le chef ou principal. Généralement, cette position est assumée par l'aîné d'une fratrie dont le père a été également l'ainé. Le reste du groupe est composé par les individus possédant des liens généalogiques avec le principal, ainsi que leurs conjoints.

6. Pour qu'un mitote soit considéré comme réussi, il faut que le chanteur et ceux qui guident les danses arrivent à faire danser le plus grand nombre de personnes et à les motiver pendant toute la nuit. Pour une description détaillée des mitote náyeri, voir Preuss (1998a et b ; 1912), Coyle (2001), Guzmán (2002), Valdovinos (2002) et Alcocer (2003).

7. Dix chants sont exécutés, l'un après l'autre, tout au long de la nuit. Chacun d'eux dure approximativement une heure.

8. Le parallélisme entre le maïs et le cerf, sur lequel l'action rituelle insiste à travers sa mise en scène, ne coïncide certainement pas avec la place que ces éléments occupent dans la vie quotidienne des Náyeri : en effet, le maïs et l'agriculture sont beaucoup plus importants que le cerf et la chasse.

9. Le préfixe ta- est le pronom possessif de la première personne du pluriel. Les racines ha et tyí se traduisent respectivement par " frère aîné » et " tante » (mère classificatoire). La composition des noms 
des divinités correspond donc à des termes de parenté qui prennent, comme ego, les Náyeri. Tahá, " Notre Frère Aîné ", et Tatyí, " Notre Mère ", correspondent, après Tayau, " Notre Père ", aux divinités principales du panthéon náyeri. Les trois sont étroitement liés à des éléments saillants de la religion náyeri (respectivement le cerf, le maïs et le soleil), mais aussi à des entités associées au panthéon catholique (respectivement Saint Michel, la Vierge Marie et Christ). Dans cette analyse, je ne considère que les deux premières divinités, car la troisième (Tayau) ne fait l'objet que de certaines mentions au cours des mitote.

10. Ce long parcours s'avère indispensable pour la conservation du savoir-faire rituel, ainsi que pour la transmission des chants dans le cas des participants masculins.

11. En cora, ces charges reçoivent les noms de Ha'atsikan tï baïre et Teih tï baïre, littéralement " celui qui aide Ha'atsikan » et " celle qui aide Teih ».

12. Ici, il semble indispensable d'établir une distinction entre « relation » et " interaction ». Pour cela, je reprends la proposition de Berthomé (2006) qui définit une " relation » comme le rapport intentionnel et virtuel entre deux ou plusieurs agents qui est spécifié par des propriétés formelles, et une " interaction » comme l'ensemble des perceptions, gestes, paroles et émotions, c'est-à-dire la mise en rapport concrète de deux ou plusieurs agents. Ainsi, chaque type de relation recouvre une gamme d'interactions pertinentes qui l'actualisent en retour. Dans cette étude, puisque le maïs et le cerf sont traités comme des agents, la relation « agriculture », par exemple, inclurait les interactions « sélection des grains ", " semailles » et « récolte », telles qu'elles sont mises en scène dans le rituel.

13. Puisque la présence de ce gibier est considérée comme très souhaitable, il arrive qu'un chasseur parte à la chasse des mois à l'avance. Dans ce cas, la majeure partie de la viande rapportée sera séchée afin de pouvoir être utilisée à l'occasion du rituel et seule une petite partie pourra être consommée par la famille du chasseur, à condition de mettre à l'abri tous les restes. Ces déchets seront rassemblés avec ceux obtenus après la consommation rituelle. L'ensemble sera ultérieurement déposé dans une caverne située dans la montagne dont les esprits sont considérés comme les protecteurs de l'espace rituel. Un traitement similaire est accordé au maïs qui est utilisé dans les mitote : aucune partie des grains ne doit être négligée ou laissée à la portée des animaux ; les déchets étant également déposés à la fin du rituel dans la grotte mentionnée plus haut.

14. Chacun des mitote est en relation avec un moment précis du cycle agricole et le maïs employé dans le rituel est donc mis en scène en accord avec cette phase du cycle : soit comme grains évoquant les semailles, soit comme épis frais de la nouvelle récolte, soit encore comme épis secs destinés au stockage.

15. Le cerf étant considéré comme le gardien du maïs et comme celui qui s'occupe de lui fournir tout ce dont il a besoin, la possession de la gourde d'eau servant à asperger les participants du rituel indique que son détenteur agit en tant que gardien du maïs, donc en tant que cerf.

16. Dans cet article, le terme " personnification » fait référence à l'action de montrer sous les traits d'une personne une entité autre. $\mathrm{Si}$, dans le cas ethnographique qui nous occupe, cette entité autre est une divinité, cela ne veut pas dire qu'il s'agisse d'un cas de possession où la personnification et la divinité sont confondues, comme il arrive dans d'autres situations rituelles (Gell 1998, pp. 150-153). Ainsi, en dépit du fait que les personnes qui assument la personnification des divinités sont appelées du nom de ces dernières, tous les participants du rituel s'accordent à dire qu'il ne s'agit pas de la divinité elle-même. Toutefois, cette personnification est spéciale et elle doit être traitée de manière particulière, au moins pendant son intervention dans les actions rituelles. De ce fait, on peut dire que les personnifications dont on traite semblent être des exemples typiques de "pièges à pensée ") (Smith 1979, pp. 139-170).

17. Dans ce sens, le chanteur apparaît comme un médiateur entre les personnifications visibles des divinités et leurs personnifications invisibles.

18. Ce type de dédoublements par l'action des chants est un phénomène courant dans les chants chamaniques. Severi (1996, p. 239) donne un exemple très intéressant d'un chant thérapeutique cuna où le chamane se construit lui-même une identité complexe par un procédé similaire que l'auteur décrit comme une "construction en abîme de l'énonciateur 》. Il définit ailleurs ce phénomène comme « réflexivité » (Severi 2002). 
19. Comme dans certains cas amazoniens (Descola 1986, p. 93), la mythologie náyeri conçoit un temps où les discontinuités physiques n'étaient pas pertinentes, car tous les êtres de la nature avaient une apparence humaine. Dans ce texte, cependant, je ne pars pas de la croyance. Je m'intéresse plutôt à la manière dont la discontinuité physique entre les humains et ces autres entités est traitée au sein des interactions rituelles.

20. En raison du manque d'espace, je n'inclus pas ici la transcription des chants de mitote. Toutefois, le lecteur intéressé peut trouver des exemples dans Preuss (1998d ; 1912).

21. Ce processus est en fait la technique culinaire utilisée pour la préparation du cerf chassé qui a lieu la veille des danses des mitote.

22. Cela dépend du moment du cycle agricole auquel correspond le mitote.

23. Le chant et les actions rituelles conduisent à attribuer deux identités à l'« enfant » de Teih : une humaine, l'autre de maïs.

24. L'analyse des extraits précédents permet d'aborder une question qui apparaît de manière récurrente dans les interprétations anthropologiques sur ce type de matériel : l'identification des personnages participant à des processus agricoles et cynégétiques avec des entités généralement représentées par des planètes et des étoiles. Dans ces analyses, on a beaucoup discuté de la possibilité d'établir des analogies entre les couples chasseur/proie ou agriculteur/maïs et les couples inspirés des observations astrales comme celui de l'étoile du matin/étoile du soir, en ce qui concerne la planète Vénus, et le couple soleil diurne/soleil nocturne, qui apparaissent aussi comme les faces opposées d'une même entité. Dans cette littérature anthropologique, il existe une tendance à envisager la relation entre aspects opposés d'un même dieu astral comme un modèle pour comprendre des pratiques et des croyances religieuses, allant de l'agriculture jusqu'au sacrifice, en passant par les rites propitiatoires de pluie et la chasse au cerf. Pour plus de détails sur cette perspective voir Preuss $(1998 \mathrm{c} ; 1912 ; 1929)$ et Neurath (2004).

25. Dans le mitote de juin, l'accent est mis sur l'activité agricole, tandis que dans les deux autres c'est le processus culinaire qui est mis en scène. Toutefois, le traitement culinaire donné à voir dans le rituel est conçu comme une métaphore des techniques et des conditions nécessaires pour le travail agricole.

26. Dans la mythologie, par exemple, Tahá, « Notre Frère Aîné », est considéré autant comme un chasseur que comme un cerf et Tatyí, " Notre Mère », est conçue comme une femme qui se moud elle-même car elle est aussi maïs (Preuss 1912).

27. Cette approche permet d'expliquer le fait que le maïs soit considéré autant comme une mère qui nourrit les hommes que comme une petite fille qui demande des soins.

28. D'après Robertson Smith $(1969$, p. 345), " [...] the fiundamental idea of sacrifice is not that of a sacred tribute, but of communion between the god and his worshippers by joint participation in the living flesh and blood of a sacred victim ".

29. Descola $(2005, \mathrm{pp} .22,35,126)$ a indiqué à plusieurs occasions l'importance des liens de ce type établis entre les hommes et les entités susceptibles de devenir leur nourriture.

\section{RÉFÉRENCES CITÉES}

\section{Alcocer Paulina}

2003 «El mitote parental de la chicharra (Metineita Tsikiri) en Chuísete'e », in Jesús Jáuregui et Johannes Neurath (éds), Flechadores de estrellas. Nuevas aportaciones a la etnología de coras y huicholes, Instituto Nacional de Antropología e Historia/CONACULTA/Universidad de Guadalajara, Mexico, pp. 181-206. 


\section{Alvarado Neira}

2004 Atar la vida, trozar la muerte. El sistema ritual de los mexicaneros de Durango, Universidad Michoacana de San Nicolás de Hidalgo, Exconvento de Tiripetío, Morelia.

\section{BERTHOMÉ François}

2006 Être affecté. Relations, interactions et émotions dans quelques rituels mélanésiens, mémoire de master 2, École des Hautes Études en Sciences Sociales, Paris.

CoYLe Phillip E.

2001 Náyeri history, politics, and violence, University of Arizona Press, Tucson.

Descola Philippe

1986 La nature domestique. Symbolisme et praxis dans l'écologie des Achuar, Éditions de la Maison des Sciences de l'Homme, Paris.

2005 Par-delà nature et culture, Gallimard, Paris

\section{GeLl Alfred}

1998 "The distributed person", in Art and agency. An anthropological theory, Clarendon Press, Oxford, pp. 96-154.

GonZÁlez Ramos Gildardo

1972 Los coras, Secretaría de Educación Pública/Instituto Nacional Indigenista, Mexico.

\section{GuZmán Adriana}

2002 Mitote $y$ universo cora, Instituto Nacional de Antropología e Historia/Universidad de Guadalajara, Mexico.

Houseman Michael et Carlo Severi

1994 Naven ou le donner à voir: Essai d'interprétation de l'action rituelle, CNRS Éditions/Éditions de la Maison des Sciences de l'Homme, Paris.

\section{Hubert Henri et Marcel Mauss}

1968a "Essai sur la nature et la fonction du sacrifice », in Euvres de Marcel Mauss. 1. Les fonctions sociales du sacré, Les Éditions de Minuit, Paris, pp. 193-354 [1899].

1968b "Introduction à l'analyse de quelques phénomènes religieux 》, in CEuvres de Marcel Mauss. 1. Les fonctions sociales du sacré, Les Éditions de Minuit, Paris, pp. 3-65 [1906].

Neurath Johannes

2004 «El doble personaje del planeta Venus en las religiones indígenas del Gran Nayar: mitología, ritual agrícola y sacrificio », Journal de la Société des Américanistes, 90 (1), pp. 93-118.

Preuss Konrad Theodor

1912 Die Nayarit-Expedition. Textaufnahmen und Beobachtungen unter Mexikanischen Indianern. 1. Die Religion der Cora-Indianer in Texten nebst Wörterbuch Cora-Deutsch, G. B. Teubner, Liepzig.

1929 «Das Frühlingsfest im alten Mexiko und bei den Mandan Indianern der Vereinigten Staaten von Nordamerika », Donum Natalictum Schrijnen. Verza- 
meling van opstellen door oud-leerlingen en bevriende vakgenooten opgedragen aan Mgr: Prof. Dr. Jos. Schrijnen bij Gelegenheid van zijn zestigsten verjaardag 3 Mei 1929, Imprimerie Durand, Chartres, pp. 825-837.

1998a «La danza mitote de los indios coras », in Jesús Jáuregui y Johannes Neurath (éds), Fiesta, literatura y magia en el Nayarit. Ensayos sobre coras, huicholes y mexicaneros de Konrad Theodor Preuss, Instituto Nacional Indigenista/Centro Francés de Estudios Mexicanos y Centroamericanos, Mexico, pp. 119-126 [1906].

1998b "Dos cantos del mitote de la Chicharra », in Jesús Jáuregui y Johannes Neurath (éds), Fiesta, literatura y magia en el Nayarit. Ensayos sobre coras, huicholes y mexicaneros de Konrad Theodor Preuss, Instituto Nacional Indigenista/Centro Francés de Estudios Mexicanos y Centroamericanos, Mexico, pp. 139-152 [1906].

1998c «La religión astral mexicana en tiempos prehispánicos y en la actualidad », in Jesús Jáuregui y Johannes Neurath (éds), Fiesta, literatura y magia en el Nayarit. Ensayos sobre coras, huicholes y mexicaneros de Konrad Theodor Preuss, Instituto Nacional Indigenista/Centro Francés de Estudios Mexicanos y Centroamericanos, Mexico, pp. 317-322 [1908].

1998d «Los cantos religiosos y los mitos de algunas tribus de la sierra Madre Occidental », in Jesús Jáuregui y Johannes Neurath (éds), Fiesta, literatura y magia en el Nayarit. Ensayos sobre coras, huicholes y mexicaneros de Konrad Theodor Preuss, Instituto Nacional Indigenista/Centro Francés de Estudios Mexicanos y Centroamericanos, Mexico, pp. 265-287 [1908].

ROBERTSON SMITH William

1969 Lectures on the religion of the Semites: the fundamental institutions, KTAV Publishing House, New York [1889].

\section{Severi Carlo}

1996 La memoria ritual. Locura e imagen del blanco en una tradición chamánica amerindia, Ediciones Abya-Yala, Quito.

2002 "Memory, reflexivity, and belief. Reflections on the ritual use of language ", Social Anthropology, 10 (1), pp. 23-40.

\section{SMith Pierre}

1979 «Aspects de l'organisation des rites », in Pierre Smith et Michel Izard (éds), La fonction symbolique, Gallimard, Paris, pp. 139-170.

VALDovinos Margarita

2002 Los cargos del pueblo de Jesús María : uma réplica de la cosmovisión cora, mémoire de maîtrise, Escuela Nacional de Antropología e Historia, Mexico. 